\title{
High-rate Information-Lossless Linear Dispersion STBCs from Group Algebra
}

\author{
Kiran. T \\ ECE Department \\ Indian Institute of Science \\ Bangalore-560012, INDIA \\ Email: kirant@protocol.ece.iisc.ernet.in
}

\author{
B. Sundar Rajan \\ ECE Department \\ Indian Institute of Science \\ Bangalore-560012, INDIA \\ Email: bsrajan@ece.iisc.ernet.in
}

\begin{abstract}
For multiple-input multiple-output (MIMO) channels, at high spectral efficiencies, space-time block codes (STBCs) must be designed to maximize the mutual information between the transmit and receive signals. In an uncoded scheme (spatial multiplexing or V-BLAST), it is well-known that the Gaussian input distribution maximizes the mutual information. Hassibi and Hochwald introduced a linear dispersion (LD) framework for designing space-time codes, wherein, any transmit codeword is a linear combination of a fixed set of matrices called the weight matrices. A LD space-time block code is said to be information-lossless if it does not disturb the maximum mutual information between the transmit and receive signals. In other words, a MIMO scheme using information-lossless LD spacetime block codes has the same capacity as the uncoded scheme. Through computer search, information-lossless LD codes with better diversity compared to uncoded system were found by Hassibi et al., and also by Heath et al. In this paper, we give a general algebraic construction of high-rate informationlossless STBCs, both square and rectangular, by restricting the weight matrices to those which form a finite group under matrix multiplication.
\end{abstract}

\section{INTRODUCTION}

A Linear Dispersion (LD) Space-Time Block Code (STBC) $\mathcal{C}$ [1] over a signal set $\mathcal{S}$, is a finite set of $n \times l$ matrices $(n \leq l)$, where any codeword matrix belonging to the code $\mathcal{C}$ is of the form

$$
\mathbf{S}\left(x_{1}, x_{2}, \ldots, x_{Q}\right)=\sum_{i=1}^{Q}\left(x_{i} \mathbf{A}_{i}+x_{i}^{*} \mathbf{B}_{i}\right),
$$

where $\mathbf{A}_{i}$ and $\mathbf{B}_{i}$ are fixed $n \times l$ complex matrices defining the LD code, $x_{1}, x_{2} \ldots, x_{Q}$ are complex scalars taking values from the signal set $\mathcal{S}$ and $x_{i}^{*}$ denotes the complex conjugate of $x_{i}$. A subclass of LD codes is the purely complex LDSTBCs, where any matrix $\mathbf{S}\left(x_{1}, x_{2}, \ldots, x_{Q}\right)$ is a complex linear combination of $\left\{\mathbf{A}_{1}, \mathbf{A}_{2}, \ldots, \mathbf{A}_{Q}\right\}$ only, i.e., the matrices $\mathbf{B}_{i}$ are all chosen to be zero matrix. We call the set $\left\{\mathbf{A}_{1}, \mathbf{A}_{2}, \ldots, \mathbf{A}_{Q}\right\}$ as dispersion matrices or weight matrices. Such complex LD-STBCs have been considered in [2] also. The rate of this code is $Q / l$ complex symbols per channel use $(Q / l$-scu).

From the pair-wise error probability point of view, it is well known that the rank criteria and the determinant criteria are

This work was partly funded by the IISc-DRDO program on Advanced Research in Mathematical Engineering through a grant to B.S. Rajan. guidelines for designing good STBCs. In [1], Hassibi et al. show that at high spectral efficiencies, while the rank criteria plays a secondary role, it is the mutual information between the transmit and receive signals that is primary with regard to both capacity as well as performance. Hence, a necessary condition for achieving capacity of a multiple-input multipleoutput (MIMO) channel is to design STBCs that maximize the mutual information. A LD-STBC is said to be informationlossless if it does not disturb the maximum mutual information between the transmit and receive signals. In other words, a MIMO scheme using information-lossless LD-STBC has the same capacity as the uncoded scheme. To make this precise, we introduce the MIMO channel model.

Let $n_{t}$ and $n_{r}$ be the number of transmit and receive antennas respectively. For an uncoded system, let $\mathbf{x} \in \mathbb{C}^{n_{t} \times 1}$ denote the vector of transmitted signals by all $n_{t}$ transmit antennas in one channel use, and let $\mathbf{y} \in \mathbb{C}^{n_{r} \times 1}$ denote the corresponding received vector. They are related by

$$
\mathbf{y}=\sqrt{\frac{\rho}{n_{t}}} \mathbf{H} \mathbf{x}+\mathbf{w}
$$

where $\rho$ is the signal to noise ratio at each receive antenna, $\mathbf{H} \in \mathbb{C}^{n_{r} \times n_{t}}$ is the channel matrix and $\mathbf{w} \in \mathbb{C}^{n_{r} \times n_{t}}$ is the additive noise. Both $\mathbf{H}$ and $\mathbf{w}$ have entries that are i.i.d, complex-Gaussian with zero mean and unit variance. It is assumed that $\mathbf{H}$ is perfectly known at the receiver, in which case the channel capacity is given by [1], [3]

$$
C\left(\rho, n_{t}, n_{r}\right)=E_{\mathbf{H}} \log _{2}\left(\operatorname{det}\left(\mathbf{I}_{n_{r}}+\frac{\rho}{n_{t}} \mathbf{H H}^{H}\right)\right) .
$$

The above expression is the capacity for the uncoded MIMO system, when an independently chosen vector $\mathbf{x}$ is transmitted for every channel use. For quasi-static fading scenario, the channel matrix will not change for successive $l$ channel uses, in which case sufficient redundancy can be introduced in the successive transmit vectors to improve the performance (in terms of probability of error) of the code. For such a case, we can write the counter part of (1) as

$$
\mathbf{Y}=\sqrt{\frac{\rho}{n_{t}}} \mathbf{H S}+\mathbf{W}
$$

where $\mathbf{S}$ is a $n_{t} \times l$ space-time codeword, $\mathbf{W}$ is the $n_{r} \times l$ complex-Gaussian additive noise matrix and $\mathbf{Y} \in \mathbb{C}^{n_{r} \times l}$ is 
the received signal matrix. For this model, mutual information between the transmitted matrix and the received matrix is calculated by using an equivalent channel model (see [2], [3], [4] for details)

$$
\operatorname{vec}(\mathbf{Y})=\sqrt{\frac{\rho}{n_{t}}} \mathcal{H} \Phi\left[\begin{array}{llll}
x_{1} & x_{2} & \cdots & x_{Q}
\end{array}\right]^{T}+\operatorname{vec}(\mathbf{W})
$$

where $\operatorname{vec}(\mathbf{Y})$ is the $n_{r} l \times 1$ matrix obtained by stacking columns of $\mathbf{Y}$ one below the other, $\mathcal{H}=$ $\operatorname{diag}(\mathbf{H}, \mathbf{H}, \ldots, \mathbf{H})$ is a $n_{r} l \times n_{t} l$ block diagonal matrix, $x_{1}, x_{2}, \ldots, x_{Q} \in \mathcal{S}$ such that $\mathbf{S}=\sum_{i=1}^{Q} x_{i} \mathbf{A}_{i}$ and $\Phi=$ $\left[\operatorname{vec}\left(\mathbf{A}_{1}\right) \operatorname{vec}\left(\mathbf{A}_{2}\right) \cdots \operatorname{vec}\left(\mathbf{A}_{Q}\right)\right]$ is a $n_{t} l \times Q$ matrix (called precoding matrix in [3]). Observe that the matrix $\Phi$ is only dependent on the weight matrices $\left\{\mathbf{A}_{1}, \mathbf{A}_{2}, \ldots, \mathbf{A}_{Q}\right\}$. To be precise, $j$-th column of $\Phi$ is equal to $\operatorname{vec}\left(\mathbf{A}_{j}\right)$. Now, the maximum mutual information between the transmitted signal vector $\left[x_{1}, x_{2}, \ldots, x_{Q}\right]^{T}$ and the received vector $\operatorname{vec}(\mathbf{Y})$ in this equivalent channel is given by

$$
\begin{aligned}
C_{S T B C}\left(\rho, n_{t}, n_{r}\right) & =\frac{1}{l} E_{\mathbf{H}} \log _{2}\left(\operatorname{det}\left(\mathbf{I}_{n_{r} l}+\frac{\rho}{n_{t}} \mathcal{H} \Phi \Phi^{H} \mathcal{H}^{H}\right)\right) \\
& \leq C\left(\rho, n_{t}, n_{r}\right) .
\end{aligned}
$$

Definition 1 ([2], [3], [4]): A space-time block code $\mathcal{C}$ is said to be information-lossless if $C_{S T B C}\left(\rho, n_{t}, n_{r}\right)=$ $C\left(\rho, n_{t}, n_{r}\right)$.

When $Q \geq n_{t} l, C_{S T B C}\left(\rho, n_{t}, n_{r}\right)=C\left(\rho, n_{t}, n_{r}\right)$ if $\Phi \Phi^{H}=\mathbf{I}_{n_{t} l}$ (see [2]). Further, when $Q=n_{t} l$, this condition in terms of weight matrices is equivalent to $\operatorname{tr}\left(\mathbf{A}_{i} \mathbf{A}_{j}^{H}\right)=\delta_{i j}$, where $\delta_{i j}=0$ if $i \neq j$, else it is equal to 1 . This condition coincides with the condition given in [4] for codes from division algebra to be information-lossless.

Both [1] and [2] resort to computer search for designing few such codes. In this paper, we take recourse to group representations to construct weight matrices which satisfy the condition $\Phi \Phi^{H}=\mathbf{I}_{n_{t} l}$. We give an algebraic construction of a class of information-lossless complex LD codes (both square as well as rectangular), where the restriction imposed is, (i) in case of square LD codes, the set of weight matrices $\left\{\mathbf{A}_{1}, \mathbf{A}_{2}, \ldots, \mathbf{A}_{Q}\right\}$ form a group under matrix multiplication and (ii) in case of rectangular LD codes, the weight matrices are derived from a set of matrices that form a group.

The space-time codes in this paper are different in the following aspects:

- While the square information-lossless codes from division algebra and TAST have rate $n_{t}$-scu, we construct arbitrary rate information-lossless codes.

- To our knowledge, the only rectangular informationlossless codes thus far, have been obtained by puncturing (deleting columns or rows of) a square TAST code [5]. In this paper, we give construction method for rectangular information-lossless codes which are not obtained from any square information-lossless code.

- In [1], for very high rates, the weight matrices obtained through computer search were not listed because the number of weight matrices were too large to be listed.
Therefore, at very high rates, space-time encoder description itself is a problem. In this paper, by restricting ourselves to LD codes based on a group algebra, we are able to describe the entire space-time code by just specifying the generators of the group. This is usually much smaller than the size of the group itself.

- In this paper, emphasis is on information-lossless property of the code, rather than the rank criteria.

\section{GROUP ALGEBRA AND ITS REPRESENTATIONS}

Let $G$ be a finite group (with $|G|$ number of elements) and let $\mathbb{C}$ denote the complex field. A group algebra $\mathbb{C}[G]$ is the set of all formal sums $\sum_{g \in G} a_{g} g, a_{g} \in \mathbb{C}$, with addition and multiplication defined as

$$
\begin{aligned}
\sum_{g \in G} a_{g} g+\sum_{g \in G} b_{g} g & =\sum_{g \in G}\left(a_{g}+b_{g}\right) g \quad \text { and } \\
\left(\sum_{g \in G} a_{g} g\right)\left(\sum_{g \in G} b_{g} g\right) & =\sum_{g \in G}\left\{\sum_{h \in G} a_{g h^{-1}} b_{h}\right\} g .
\end{aligned}
$$

A linear representation of $G$ is a homomorphism $\sigma$ from $G$ into the group of $d \times d$ invertible matrices $G L(d, \mathbb{C})$. In other words, to each element $g$ of $G$ we associate an invertible $d \times d$ matrix $\left[\sigma_{g}\right]$, and these satisfy $\left[\sigma_{g}\right]\left[\sigma_{h}\right]=\left[\sigma_{g h}\right]$ for any $g, h \in$ $G$. We call $d$, the degree of representation $\sigma$. Further, if the matrix $\left[\sigma_{g}\right]=\left(r_{i j}(g)\right)$, i.e., the $(i, j)$-th entry of matrix $\left[\sigma_{g}\right]$ is $r_{i j}(g)$, we view $r_{i j}()$ as a function from $G$ to $\mathbb{C}$. If $d$ is the degree of representation $\sigma$, then there are $d^{2}$ different functions $r_{i j}()$ corresponding to $1 \leq i, j \leq d$, called the coordinate functions which characterize $\sigma$. A representation $\sigma$ is said to be faithful if it is an isomorphism. Two representations $\sigma$ and $\tau$ are said to be equivalent (or similar) if there exists a nonsingular matrix $A$ such that $\left[\sigma_{g}\right]=A\left[\tau_{g}\right] A^{-1}$ for all $g \in G$. Every representation has an equivalent unitary representation (in which each matrix $\left[\sigma_{g}\right]$ is a unitary matrix).

A group representation can be linearly extended to give a representation of the group algebra $\mathbb{C}[G]$. Let $M_{d}(\mathbb{C})$ denote the set of all $d \times d$ complex matrices. If $\sigma$ is representation of $G$, then $\sigma: \mathbb{C}[G] \rightarrow M_{d}(\mathbb{C})$, is a $\mathbb{C}$-algebra homomorphism such that $\sigma\left(\sum_{g \in G} a_{g} g\right)=\sum_{g \in G} a_{g}\left[\sigma_{g}\right]$. If the degree of $\sigma$ is $d$, then we can get a rate $|G| / d$, linear STBC using weight matrices $\{[\sigma(g)], g \in G\}$ and a finite signal set $\mathcal{S} \subset \mathbb{C}$.

A representation $\sigma$ is said to be reducible if $\left[\sigma_{g}\right]$ is similar to a block-diagonal matrix for all $g \in G$, else it is called an irreducible representation. If a representation $\sigma$ is reducible, the blocks that appear in the block-diagonal matrix are called irreducible constituents of $\sigma$. Following lemma will be used for constructing information-lossless STBCs in Sections III and IV.

Lemma 1 (Schur's Lemma [6], [7]): If $\left[\rho_{g}\right]=\left(r_{i_{1} j_{1}}(g)\right)$ and $\left[\sigma_{g}\right]=\left(s_{i_{2} j_{2}}(g)\right)$ are two irreducible representations of $G$, then:

1) if $\rho$ and $\sigma$ are not isomorphic, then

$$
\frac{1}{|G|} \sum_{g \in G} s_{i_{2} j_{2}}\left(g^{-1}\right) r_{i_{1} j_{1}}(g)=0
$$


for arbitrary $i_{1}, i_{2}, j_{1}, j_{2}$.

2) if $\rho=\sigma$ and degree of $\rho$ is $d$, then

$$
\frac{1}{|G|} \sum_{g \in G} r_{i_{2} j_{2}}\left(g^{-1}\right) r_{i_{1} j_{1}}(g)=\frac{1}{d} \delta_{i_{2} i_{1}} \delta_{j_{2} j_{1}}
$$

where $\delta_{i_{2} i_{1}}$ is equal to 1 if $i_{1}=i_{2}$ and 0 otherwise.

Suppose that the matrices $\left(r_{i j}(g)\right)$ are unitary (recall that every representation has an equivalent unitary representation), we have $r_{i j}\left(g^{-1}\right)=r_{j i}(g)^{*}$ and (4) reduces to

$$
\frac{1}{|G|} \sum_{g \in G} r_{j_{2} i_{2}}(g)^{*} r_{i_{1} j_{1}}(g)=\frac{1}{d} \delta_{i_{2} i_{1}} \delta_{j_{2} j_{1}} .
$$

Further, if $\rho^{(1)}, \rho^{(2)}, \ldots, \rho^{(K)}$ are all the distinct nonequivalent representations of degree $d_{1}, d_{2}, \ldots, d_{K}$ respectively, and if $r_{i j}^{(k)}(), 1 \leq k \leq K, 1 \leq i, j \leq d_{k}$ are the corresponding coordinate functions, it can be shown that $\sum_{i=1}^{K} d_{i}^{2}=|G|$. If $\mathcal{T}_{G}$ is a $|G| \times|G|$ matrix with each row defined by a coordinate function, equal to $\left(r_{i j}^{(k)}\left(g_{1}\right), r_{i j}^{(k)}\left(g_{2}\right), \cdots, r_{i j}^{(k)}\left(g_{|G|}\right)\right)$, then it follows from the above lemma that $\mathcal{T}_{G} \mathcal{T}_{G}^{H}=D$, where $D$ is a $|G| \times|G|$, full rank diagonal matrix. This means that the rows of $\mathcal{T}_{G}$ form an orthogonal basis of $|G|$-dimensional complex vector space, a property that will be useful in the next section.

\section{SQuARE INFORMATION-LOSSLESS CODES $\left(n_{t}=l\right)$}

Suppose we wish to design a linear, information-lossless square STBC for $n_{t}$ transmit antennas. This involves finding a finite set of $n_{t} \times n_{t}$ matrices $\left\{\mathbf{A}_{1}, \mathbf{A}_{2}, \ldots, \mathbf{A}_{Q}\right\}$, satisfying the property $\Phi \Phi^{H}=\mathbf{I}$, where $\Phi=$ $\left[\operatorname{vec}\left(\mathbf{A}_{1}\right) \operatorname{vec}\left(\mathbf{A}_{2}\right) \cdots \operatorname{vec}\left(\mathbf{A}_{Q}\right)\right]$. We choose a finite group $G$ with an irreducible representation of degree $d=n_{t}$ (say $\sigma$ ). The set of weight matrices are then equal to $\left\{\mathbf{A}_{g}=\sqrt{\frac{n_{t}}{|G|}}\left[\sigma_{g}\right] \mid g \in G\right\}$, and any codeword matrix is equal to $\sum_{g \in G} a_{g} \mathbf{A}_{g}$ for some $a_{g} \in \mathcal{S}$.

Theorem 1: With the choice of weight matrices as above, a linear STBC based on a group algebra $\mathbb{C}[G]$ is informationlossless if the order of group satisfies $|G| \geq n_{t}^{2}>1$.

In the rest of this paper, we mostly concentrate on the simplest non-Abelian group (dihedral group) to demonstrate the relevance of group algebra framework in tackling informationlossless code design issues in MIMO.

Dihedral group $D_{m}$ : This is the group of rotations and reflections of the plane which preserve a regular polygon with $m$ vertices. If we denote by $r$ the rotation by an angle $2 \pi / m$ and if $s$ is any one of the reflections, we have: $D_{m}=$ $\left\langle r, s \mid r^{m}=s^{2}=1, s r s=r^{-1}\right\rangle$. Each element of $D_{m}$ can be written uniquely, either as $r^{k}$, or as $s r^{k}$, with $0 \leq k \leq$ $m-1$. For $m$ odd, there are two irreducible representations of degree 1 given by $\sigma_{+}(r)=\sigma_{+}(s)=1$ and $\sigma_{-}(r)=$ $1=-\sigma_{-}(s)$, where as for even $m$ there are four degree 1 irreducible representations given by $\sigma_{++}(r)=\sigma_{++}(s)=1$, $\sigma_{+-}(r)=1=-\sigma_{+-}(s),-\sigma_{-+}(r)=1=\sigma_{-+}(s)$ and $\sigma_{--}(r)=-1=\sigma_{--}(s)$. Consider the map

$\rho^{(h)}\left(r^{k}\right)=\left[\begin{array}{cc}\omega_{m}^{h k} & 0 \\ 0 & \omega_{m}^{-h k}\end{array}\right], \rho^{(h)}\left(s r^{k}\right)=\left[\begin{array}{cc}0 & \omega_{m}^{-h k} \\ \omega_{m}^{h k} & 0\end{array}\right]$, where $\omega_{m}=\exp (2 \pi j / m)$. This map is a degree 2 irreducible representation of $D_{m}$ for any $0<h<m$, but the total number of non-equivalent irreducible representations is equal to $(m / 2)-1$ (corresponding to $1 \leq h \leq(m / 2)-1)$ when $m$ is even and $(m-1) / 2$ (corresponding to $1 \leq h \leq$ $(m-1) / 2)$ when $m$ is odd. Further, not all of these are faithful representations. It can be shown that these non-equivalent irreducible representations are faithful only if $\operatorname{gcd}(h, m)=1$. In the rest of this section, we consider only faithful irreducible representations.

Example 1: Let us consider the symmetry group of a square, $D_{4}=\left\{1, r, r^{2}, r^{3}, s, s r, s r^{2}, s r^{3}\right\}$. This group has a unique irreducible representation (up to equivalence) of degree 2 given by

$$
\left[\sigma_{r}\right]=\left[\begin{array}{rr}
j & 0 \\
0 & -j
\end{array}\right] \quad \text { and } \quad\left[\sigma_{s}\right]=\left[\begin{array}{ll}
0 & 1 \\
1 & 0
\end{array}\right],
$$

where $j=\sqrt{-1}$. The corresponding set of weight matrices based on this group is $\left\{\mathbf{A}_{s^{i} r^{j}}=\frac{1}{2}\left[\sigma_{s^{i} r^{j}}\right]\right\}$, and the corresponding precoding matrix is

$$
\Phi_{D_{4}}=\frac{1}{2}\left[\begin{array}{rrrrrrrr}
1 & j & -1 & -j & 0 & 0 & 0 & 0 \\
0 & 0 & 0 & 0 & 1 & j & -1 & -j \\
0 & 0 & 0 & 0 & 1 & -j & -1 & j \\
1 & -j & -1 & j & 0 & 0 & 0 & 0
\end{array}\right] .
$$

Clearly $\Phi_{D_{4}} \Phi_{D_{4}}^{H}=\mathbf{I}_{4}$. Using these weight matrices, we get a rate 4 -scu, information-lossless code where every codeword matrix is of the form $\sum_{s^{i} r^{j} \in D_{4}} a_{s^{i} r^{j}} \mathbf{A}_{s^{i} r^{j}}$ for some $a_{s^{i} r^{j}} \in$ $\mathcal{S}$.

Observe that in the case of $D_{4}$, the representation is such that, if $\mathbf{A}$ is a weight matrix then so is $-\mathbf{A}$. This is not a desirable property for constructing linear dispersion STBCs because, two different linear combinations might produce the same codeword matrix. This will invariably cause decoding errors since there is no unique mapping between the set of complex values from $\mathcal{S}$ and the codeword matrices. In order to avoid this anomaly, we use multiple signal sets and give a general condition that these signal sets should satisfy for getting a unique mapping between the set of complex values and the codeword matrices.

Let $G=\left\{g_{1}, g_{2}, \ldots, g_{|G|}\right\}$ be a group and let $\rho^{(h)}$ be a degree $d$ (greater than 1) irreducible representation of $G$. Any codeword matrix based on this group algebra is a $d \times d$ matrix of the form $\sum_{g_{k} \in G} a_{g_{k}}\left[\rho^{(h)}\left(g_{k}\right)\right]$. Let the complex variable $a_{g_{k}}$ take values from signal set $\mathcal{S}_{g_{k}}$, a finite subset of $\mathbb{C}$ for each $g_{k} \in G$. Then the space-time encoder is a map from $\mathcal{S}_{g_{1}} \times \mathcal{S}_{g_{2}} \times \cdots \times \mathcal{S}_{g_{|G|}}$ to $M_{d}(\mathbb{C})$. This encoding map is not one-one if and only if $\sum_{g_{k} \in G} \Delta_{g_{k}}\left[\rho^{(h)}\left(g_{k}\right)\right]=$ $\mathbf{0}_{d}$, for any $\Delta_{g_{k}} \in \Delta \mathcal{S}_{g_{k}}=\left\{a_{g_{k}}-b_{g_{k}} \mid a_{g_{k}}, b_{g_{k}} \in\right.$ $\left.\mathcal{S}_{g_{k}}\right\}$. If $\left[\rho^{(h)}\left(g_{k}\right)\right]=\left(r_{i j}^{(h)}\left(g_{k}\right)\right)$, this condition can be alternately written as a set of $d^{2}$ simultaneous equations $\sum_{g_{k} \in G} \Delta_{g_{k}} r_{i j}^{(h)}\left(g_{k}\right)=0$ for all $1 \leq i, j \leq d$. This simply means the vector $\left(\Delta_{g_{1}}^{*}, \Delta_{g_{2}}^{*}, \ldots, \Delta_{g_{|G|}}^{*}\right)$ is an element of the subspace of $\mathbb{C}^{1 \times|G|}$, orthogonal to the row-space of $\Phi_{G}$.

Proposition 1: Let $G$ be a finite group and $\rho^{(h)}$ be a faithful, irreducible representation of $G$. If $\Phi_{G}$ is the pre- 


$$
\mathbf{A}_{g}=\sqrt{\frac{d}{|G|}}\left[\left[\rho_{g}\right]\left[\sigma_{g}\right]\right]=\sqrt{\frac{d}{|G|}}\left[\begin{array}{cccccccc}
r_{11}(g) & r_{12}(g) & \cdots & r_{1 d}(g) & s_{11}(g) & s_{12}(g) & \cdots & s_{1 d}(g) \\
r_{21}(g) & r_{22}(g) & \cdots & r_{2 d}(g) & s_{21}(g) & s_{22}(g) & \cdots & s_{2 d}(g) \\
\vdots & \vdots & \cdots & \vdots & \vdots & \vdots & \cdots & \vdots \\
r_{d 1}(g) & r_{d 2}(g) & \cdots & r_{d d}(g) & s_{d 1}(g) & s_{d 2}(g) & \cdots & s_{d d}(g)
\end{array}\right]
$$

coder for the space-time encoder based on weight matrices $\left\{\sqrt{\frac{n_{t}}{|G|}}\left[\rho^{(h)}\left(g_{i}\right)\right] \mid g_{i} \in G\right\}$ and signal sets $\mathcal{S}_{g_{i}}, g_{i} \in G$, this encoder is a one-one map if and only if $\left(\Delta_{g_{1}}^{*}, \Delta_{g_{2}}^{*}, \ldots, \Delta_{g_{|G|}}^{*}\right)$ for any $\Delta_{g_{i}} \in \Delta \mathcal{S}_{g_{i}}$, is not an element of the subspace spanned by all the rows of $\mathcal{T}_{G}$ that are not in $\Phi_{G}$.

Example 2: For the group $D_{4}$, the matrix $\mathcal{T}_{D_{4}}$ is equal to

$$
\mathcal{T}_{D_{4}}=\left[\begin{array}{rrrrrrrr}
1 & 1 & 1 & 1 & 1 & 1 & 1 & 1 \\
1 & 1 & 1 & 1 & -1 & -1 & -1 & -1 \\
1 & -1 & 1 & -1 & 1 & -1 & 1 & -1 \\
1 & -1 & 1 & -1 & -1 & 1 & -1 & 1 \\
1 & j & -1 & -j & 0 & 0 & 0 & 0 \\
0 & 0 & 0 & 0 & 1 & j & -1 & -j \\
0 & 0 & 0 & 0 & 1 & -j & -1 & j \\
1 & -j & -1 & j & 0 & 0 & 0 & 0
\end{array}\right],
$$

and the corresponding matrix $\Phi_{D_{4}}$ is given in Example 1 . Let $W$ be the vector space generated by the first four rows of $\mathcal{T}_{D_{4}}$. If $\mathcal{S}_{1}=\mathcal{S}_{r}=\mathcal{S}_{s}=\mathcal{S}_{s r}=\mathcal{S}$ and $\mathcal{S}_{r^{2}}=\mathcal{S}_{r^{3}}=\mathcal{S}_{s r^{2}}=$ $\mathcal{S}_{s r^{3}}=\hat{\mathcal{S}}$, such that $\Delta \mathcal{S} \cap \Delta \hat{\mathcal{S}}=\{0\}$, then the space-time encoder is a one-one map.

\section{RECTANGULAR INFORMATION-LOSSLESS CODES}

In Theorem 1, we constructed only square STBCs, where we essentially used a faithful irreducible representation of a dihedral group, and made use of only a part of Schur's lemma (given by (4)) to construct square information-lossless codes. But according to (3), the coordinate functions corresponding to two different irreducible representations are orthogonal. This property suggests that we can construct rectangular information-lossless STBCs by considering multiple non-equivalent irreducible representations.

Theorem 2: Let $\rho$ and $\sigma$ be two non-equivalent irreducible representations of a group, both of degree $d$ such that at least one of them is a faithful representation. If $\left[\rho_{g}\right]=\left(r_{i j}(g)\right)$ and $\left[\sigma_{g}\right]=\left(s_{i j}(g)\right)$ for all $g \in G$, let $\mathbf{A}_{g}$ be a weight matrix as given in (6) for $g \in G$. Then the rate $|G| / 2 d$ STBC obtained by using weight matrices $\left\{\mathbf{A}_{g} \mid g \in G\right\}$ is information-lossless for $n_{t}=d$ transmit antennas and quasi-static interval $l=2 d$ if $|G| \geq n_{t} l=2 d^{2}$.

Example 3: Consider the symmetry group $D_{5}$. This group has two non-equivalent degree 2 representations $\rho^{(1)}$ and $\rho^{(2)}$ and the corresponding weight matrices are of the form

or

$$
\mathbf{A}_{r k}=\sqrt{\frac{1}{5}}\left[\begin{array}{cccc}
\omega_{5}^{k} & 0 & \omega_{5}^{2 k} & 0 \\
0 & \omega_{5}^{-k} & 0 & \omega_{5}^{-2 k}
\end{array}\right]
$$

$$
\mathbf{A}_{s r k}=\sqrt{\frac{1}{5}}\left[\begin{array}{cccc}
0 & \omega_{5}^{-k} & 0 & \omega_{5}^{-2 k} \\
\omega_{5}^{k} & 0 & \omega_{5}^{2 k} & 0
\end{array}\right]
$$

where $0 \leq k \leq 4$. Using these matrices, we get a rate $5 / 2$ information-lossless STBC for 2 transmit antennas and quasistatic interval, $l=4$.
Corollary 1: Using the non-equivalent irreducible representations of dihedral group $D_{m}$ (with at least one being a faithful representation), and signal sets according to Proposition 1, we can get rate $\frac{m}{p}$-scu, rectangular information-lossless STBC for 2 transmit antenna and quasi-static interval, $l=2 p$ where $p$ is any integer $1 \leq p \leq \frac{m}{2}-1$ when $m$ is an even integer and $1 \leq p \leq(m-1) / 2$, when $m$ is an odd integer.

\section{A. STBCs derived from group algebra}

So far we only considered weight matrices which form a group under matrix multiplication, and as an application of Schur's lemma we were able to show that any group will give an information-lossless code. But the rate of these codes are very large and hence the complexity of (sphere) decoding is high. In this section, for a group $D_{m}$ with $m$ even, in order to reduce the decoding complexity at the cost of rate, we propose to remove the "negative" weight matrices $(-\mathbf{A})$ from the set of weight matrices. In case of $D_{4}$, the corresponding punctured precoding matrix is ${ }^{1}$

$$
\widehat{\Phi}_{D_{4}}=\frac{1}{\sqrt{2}}\left[\begin{array}{rrrr}
1 & j & 0 & 0 \\
0 & 0 & 1 & j \\
0 & 0 & 1 & -j \\
1 & -j & 0 & 0
\end{array}\right]
$$

and fortunately, this continues to satisfy $\widehat{\Phi}_{D_{4}} \widehat{\Phi}_{D_{4}}^{H}=\mathbf{I}_{4}$. Using these reduced set of weight matrices, we get a rate 2 -scu, information-lossless STBC for $n_{t}=l=2$ MIMO system. A nice property of this code is that, unlike square STBCs of the previous section which have a restriction on the signal sets, $\widehat{\Phi}_{D_{4}}$ is a square matrix and hence $\widehat{\Phi}_{D_{4}} \widehat{\Phi}_{D_{4}}^{H}=\mathbf{I}_{4}$ implies the reduced set of weight matrices are linearly independent over $\mathbb{C}$, which means any signal set can be used. This process of removing "negative" weight matrices and still retaining the information-lossless property of the code is not unique to $D_{4}$, but holds true for any even integer $m$.

Theorem 3: For any even integer $m>3$, if $\rho^{(h)}$ is a faithful, irreducible representation of the dihedral group $D_{m}$, then the reduced set of weight matrices $\mathbf{A}_{s^{i} r^{j}}=\sqrt{\frac{2}{m}}\left[\rho_{s^{i} r^{j}}^{(h)}\right]$ where $0 \leq i \leq 1$ and $0 \leq j \leq m / 2$ gives a rate $\frac{m}{2}$-scu information-lossless STBC for $n_{t}=l=2$ MIMO system.

Similar result can be proved for non-group algebra based rectangular codes from multiple irreducible representations of $D_{m}$ ( $m$ even). We only give an example due to space constraints.

Example 4: Using the irreducible representations $\rho^{(1)}$ and $\rho^{(3)}$ of the group $D_{8}$, the reduced set of weight matrices $\left\{\sqrt{\frac{2}{m}} \mathbf{A}_{s^{i} r^{j}}=\left[\left[\rho_{s^{i} r^{j}}^{(1)}\right]\left[\rho_{s^{i} r^{j}}^{(3)}\right]\right] \mid 0 \leq i \leq 1,0 \leq j<4\right\}$ gives a rate 2 -scu STBC for $n_{t}=2$ and $l=4$ MIMO system. The corresponding punctured precoding matrix $\widehat{\Phi}_{D_{8}}$

\footnotetext{
${ }^{1}$ Notice that the scaling factor is accordingly changed to make this unitary.
} 
is a square unitary matrix and hence there is no restriction on the signal set.

\section{Simulation Results}

In this section, we present simulation results, comparing the performance of our code against known information-lossless codes. We use the equivalent channel model explained in Section I and perfect channel knowledge is assumed at the receiver only.

In Fig.1, we present the simulation results for $n_{t}=n_{r}=$ $l=2$ system using various space-time codes and a 4-QAM constellation. The codes that we have considered are the uncoded space-time modulation with sphere decoding, LD code (refer equation (28) and Fig.4 in [1]), the code obtained through computer search by Heath (refer $\mathcal{X}_{2}$ and Fig.2 in [2]) and the rate-2 full-rank DA code (refer Example 11 in [4]). All these codes are described completely by their precoding matrices. We compare the performance of these codes with the STBC from $D_{4}$ with truncated precoding matrix $\widehat{\Phi}_{D_{4}}$. At $20 \mathrm{~dB}$ SNR, while LD code and $D_{4}$ code have similar performance, DA code and Heath's code perform much better. This is because the DA code has full-rank and the code in Heath's paper was obtained through computer search for maximizing mutual information as well as minimizing pairwise error probability as much as possible. But as pointed out in [1], rank criteria matters at high SNR regime, only when the spectral efficiency is much below channel capacity. In case of $n_{t}=2$ and $n_{r}=2$ MIMO system, the channel capacity at $20 \mathrm{~dB}$ SNR is about 11.28 -bits/sec/Hz [1]. When spectral efficiency is close to the channel capacity, information-lossless property is more important and this is evident in Fig. 2 where the performance of DA code, Heath's code and $\widehat{\Phi}_{D_{4}}$ for a 16-QAM constellation (8-bits/sec/Hz) is shown. All three codes perform equally well at $20 \mathrm{~dB}$ SNR because all these are information-lossless codes. In Fig.3, we compare the performance of the rectangular STBC given in Example 5 with the uncoded system for a 4QAM constellation. Our code performs better than the uncoded system at high SNR region.

\section{REFERENCES}

[1] B. Hassibi and B. Hochwald, "High-rate codes that are linear in space and time," IEEE Trans. Inform. Theory, vol. 48, no. 7, pp. 1804-1824, July 2002.

[2] R. Heath. Jr and A. Paulraj, "Capacity maximizing linear space-time codes," IEICE Trans. Electron., vol. E85-C, no. 3, pp. 428-435, Mar 2002.

[3] M. Damen, A. Tewfik, and J.-C. Belfiore, "A construction of a space-time code based on number theory," IEEE Trans. Inform. Theory, vol. 48, no. 3, pp. 753-760, Mar 2002.

[4] B. A. Sethuraman, B. S. Rajan, and V. Shashidhar, "Full-diversity, highrate space-time block codes from division algebras," IEEE Trans. Inform. Theory, vol. 49, no. 10, pp. 2596-2616, Oct 2003.

[5] P. Dayal and M. Varanasi, "Algebraic space-time codes that achieve maximal diversity and/or capacity optimality with low peak-to mean power ratio," submitted to IEEE Trans. Inform. Theory [Available Online] http://ece-www.colorado.edu/ṽaranasi/publicat.htm, May 2003.

[6] J.-P. Serre, Linear Representations of Finite Groups. New York: Springer-Verlag, 1977.

[7] C. Curtis and I. Reiner, Representation Theory of Finite Groups and Associative Algebras. New York: Interscience Pub. (division of John Wiley \& Sons), 1966.

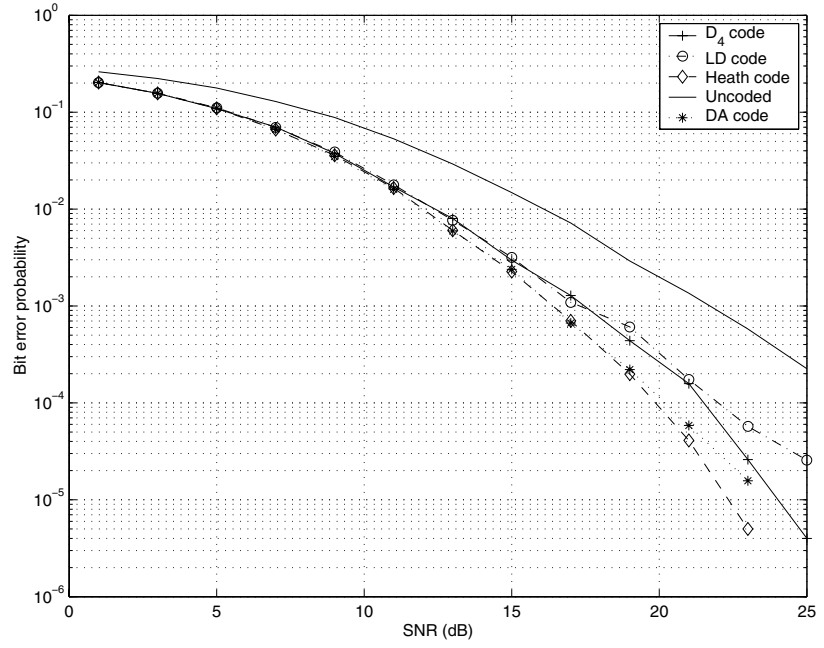

Fig. 1. Bit error rate comparison for $n_{t}=l=2,4$-bits/sec/Hz STBCs

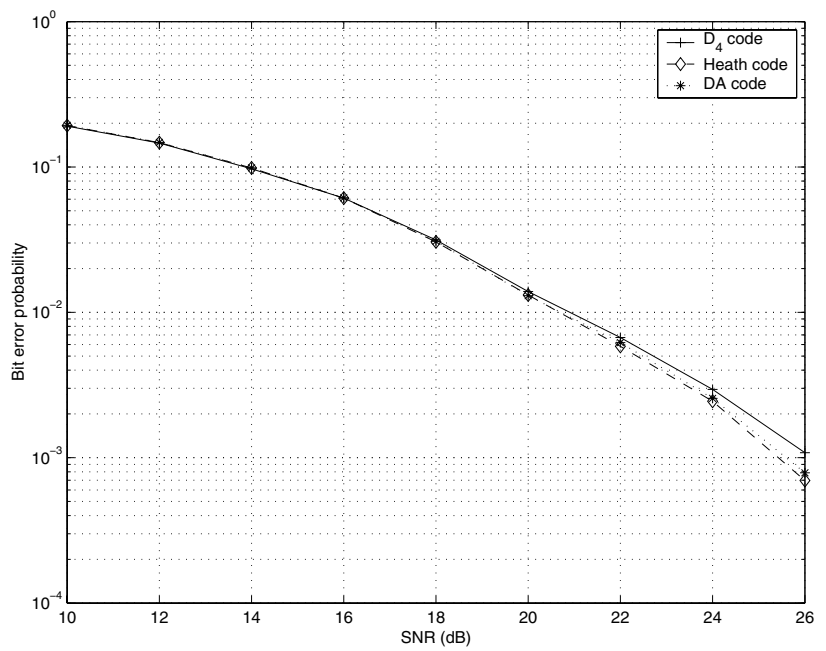

Fig. 2. Bit error rate comparison for $n_{t}=l=2,8$-bits/sec/Hz STBCs

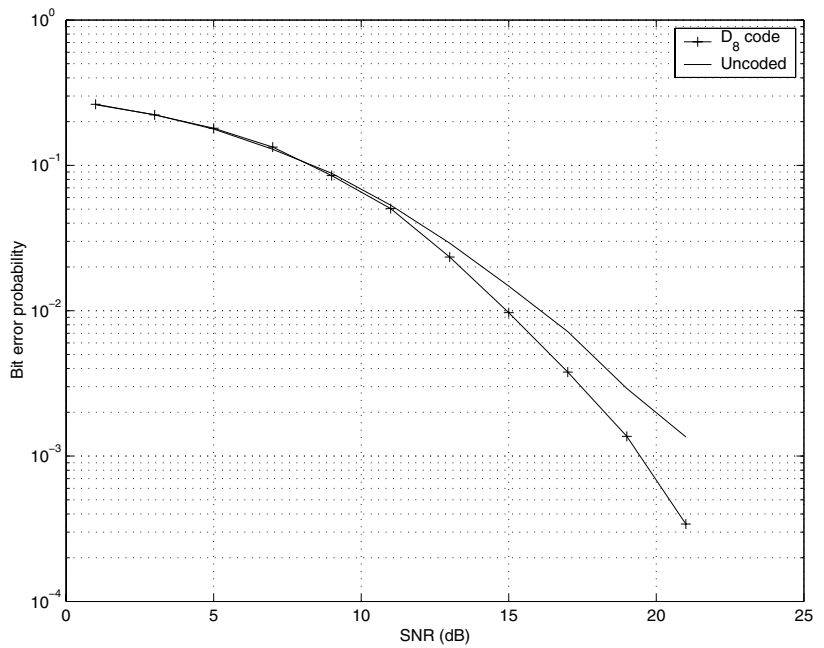

Fig. 3. Bit error rate comparison for $n_{t}=2$ and $l=4,4$-bits/sec/Hz STBCs 